\title{
Passivity-Based Control of Rotational and Translational Timoshenko Arms
}

\author{
Minoru Sasaki, ${ }^{1}$ Takeshi Ueda, ${ }^{1}$ Yshihiro Inoue, ${ }^{2}$ and Wayne J. Book ${ }^{3}$ \\ ${ }^{1}$ Department of Human and Information Systems Engineering, Gifu University, 1-1 Yanagido, Gifu 501-1193, Japan \\ ${ }^{2}$ Department of Mechanical and Systems Engineering, Gifu University, 1-1 Yanagido, Gifu 501-1193, Japan \\ ${ }^{3}$ School of Mechanical Engineering, Georgia Institute of Technology Atlanta, J. E. Love Jr. Manufacturing Building, 771 Ferst Drive, \\ GA 30332-0405, USA
}

Correspondence should be addressed to Minoru Sasaki, sasaki@gifu-u.ac.jp

Received 4 May 2011; Accepted 29 July 2011

Academic Editor: Abul Azad

Copyright () 2012 Minoru Sasaki et al. This is an open access article distributed under the Creative Commons Attribution License, which permits unrestricted use, distribution, and reproduction in any medium, provided the original work is properly cited.

It is shown that the alternate passivity-based control schemes can be designed which explicitly exploit the passivity properties of the Timoshenko model. This approach has the advantage over the conventional methods in the respect that it allows one to deal directly with the system's partial differential equations without resorting to approximations. Numerical results for the tracking control of a translational and rotational flexible Timoshenko arm are presented and compared. They verify that the proposed control schemes are effective at controlling flexible dynamical systems.

\section{Introduction}

In recent years, there has been a great deal of interest in the modeling and control of flexible arms [1-11]. This interest has been motivated by the prospect of fast, light, robot whose links, due to material characteristics, will flex under heavy loads. As a first step towards designing controllers for such robots, researchers have begun studying controllers for simple flexible links. These links, in most cases modeled as Euler-Bernoulli beams because of the small deflections involved, are often analyzed through an eigen-function series expansion of the solution to beam equation. The infinite term expansion is typically truncated after a finite number of terms and the controller designed using one of the various available linear control design techniques. Most practical controllers are designed based on models that are truncations of infinite-order transfer function models. There are several reasons for using the reduced-order models. First, these finite-order models agree very well with experimental results, assuming that an adequate number of modes have been retained. Second, there are no infinite-order transfer function models that coincide with experimental results past the first few modes. Finally, the use of a finite-order model greatly simplifies the controller design procedure. Most existing control design methods for flexible system, such as the Independent Modal Space Control, low-authority highauthority control design, frequency-weighted LQG, and positivity require modal truncation. One disadvantage of this kind of approach is that a controller, based on a truncated model, cannot guarantee stability of the closed loop system. This disadvantage arises because the model does not include the higher frequency terms. A second disadvantage is that the criteria for designing the controller for such a model are usually arbitrary and the design becomes more difficult as the truncated model order is increased.

In this paper, it is shown that the alternate passivity-based control schemes can be designed, which explicitly exploit the passivity properties of the Timoshenko model $[12,13]$. From standard nonlinear control theory, the passivity theorem tells us that any strictly passive controller with finite gain will stabilize the system in an $L_{2}$ sense. This allows the design of very simple controllers for the systems. This approach has the advantage over the conventional methods in the respect that it allows one to deal directly with the system's partial differential equations without resorting to approximations. Moreover, it is potentially applicable to the control of nonlinear flexible systems. Numerical results for the tracking control of a translational and rotational flexible Timoshenko 
arm are presented and compared. They verify that the proposed control schemes are effective at controlling flexible dynamical systems.

\section{Equation of Motions and Boundary Conditions}

2.1. Translational Arm. Figure 1 shows a sketch of a translational Timoshenko arm. The equation of motions of the arm and mobile stage are

$$
\begin{gathered}
\rho A\left(\frac{\partial^{2} y}{\partial t^{2}}+\frac{\partial^{2} W_{b}}{\partial t^{2}}\right)-\kappa G A\left(\frac{\partial^{2} y}{\partial x^{2}}-\frac{\partial \phi}{\partial x}\right)=0, \\
\rho I \frac{\partial^{2} \phi}{\partial t^{2}}-E I \frac{\partial^{2} \phi}{\partial x^{2}}-\kappa G A\left(\frac{\partial y}{\partial x}-\phi\right)=0, \\
M_{p} \frac{\partial^{2} W_{b}}{\partial t^{2}}=-\kappa G A\left(\frac{\partial y(0, t)}{\partial x}-\phi(0, t)\right)+u .
\end{gathered}
$$

with boundary conditions:

$$
\begin{aligned}
& m \frac{\partial^{2}}{\partial t^{2}}\left(y(L, t)+W_{b}\right)+\kappa G A\left(\frac{\partial y(L, t)}{\partial x}-\phi(L, t)\right)=0, \\
& I_{c} \frac{\partial^{2}}{\partial t^{2}} \phi(L, t)+E I \frac{\partial \phi(L, t)}{\partial x}=0, \\
& y(0, t)=0, \\
& \phi(0, t)=0 .
\end{aligned}
$$

Applying the Laplace transformation method with respect to $t$ to equations (1) and (2) gives

$$
\begin{gathered}
\rho A s^{2}\left(Y+W_{b}\right)-\kappa G A\left(\frac{\partial^{2} Y}{\partial x^{2}}-\frac{\partial \Theta}{\partial x}\right)=0, \\
\rho I s^{2} \Phi-E I \frac{\partial^{2} \Phi}{\partial x^{2}}-\kappa G A\left(\frac{\partial Y}{\partial x}-\Phi\right)=0, \\
M_{p} s^{2} W_{b}=-\kappa G A\left(\frac{\partial Y(0, t)}{\partial x}-\Phi(0, t)\right)+U, \\
m s^{2}\left(Y(l, s)+W_{b}\right)+\kappa G A\left(\frac{\partial y(L, s)}{\partial x}-\Phi(L, s)\right)=0, \\
I_{c} s^{2} \Phi(L, s)+E I \frac{\partial \Phi(L, s)}{\partial x}=0, \\
Y(0, s)=0, \\
\Phi(0, s)=0 .
\end{gathered}
$$

2.2. Rotational Arm. The rotational Timoshenko arm depicted in Figure 2 of total length $L$, area moment of inertia $I$, cross-sectional area $A$, density $\rho$, Young's modulus $E$, shear modulus $G$, and shear coefficient $\kappa$, is attached at one end to a payload of mass $m$ and inertia $I_{c}$ and on the other end a hub of inertia $I_{h}$, which in turn is connected to an actuator

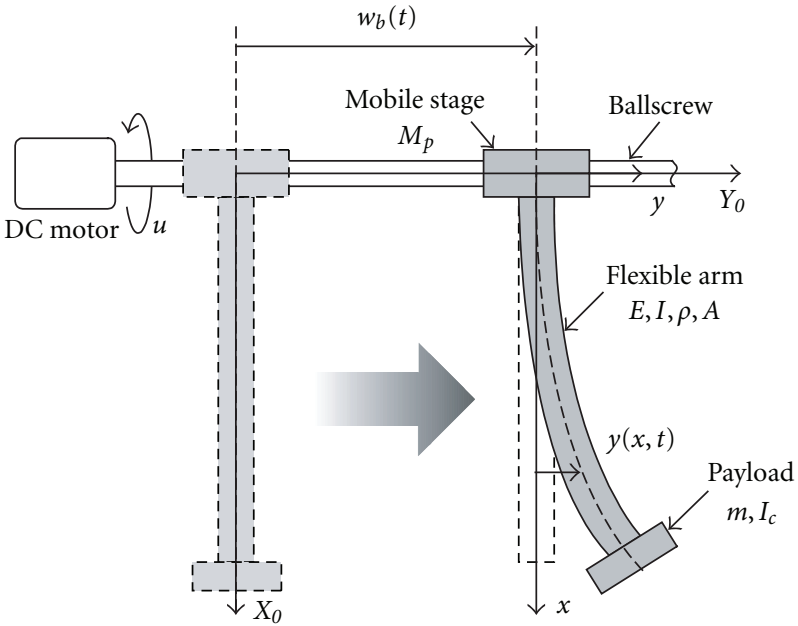

Figure 1: Translational Timoshenko arm.

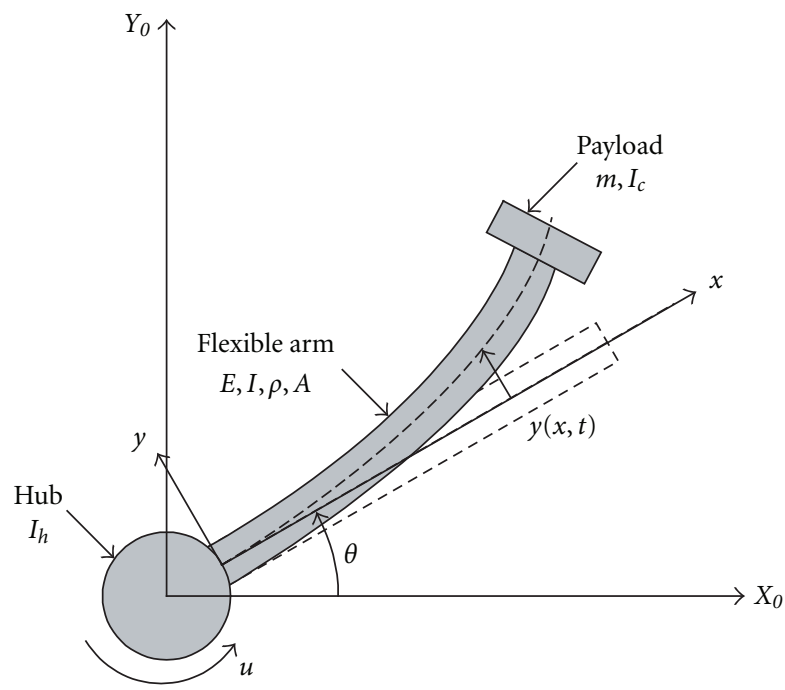

FIgURe 2: Rotational Timoshenko arm.

that supplies a torque $u$. We obtain the following equations of motion with the boundary conditions.

$$
\begin{gathered}
\rho A\left(\frac{\partial^{2} y}{\partial t^{2}}+x \frac{\partial^{2} \vartheta}{\partial t^{2}}\right)-\kappa G A\left(\frac{\partial^{2} y}{\partial x^{2}}-\frac{\partial \phi}{\partial x}\right)=0, \\
\rho I\left(\frac{\partial^{2} \phi}{\partial t^{2}}+\frac{\partial^{2} \vartheta}{\partial t^{2}}\right)-E I \frac{\partial^{2} \phi}{\partial x^{2}}-\kappa G A\left(\frac{\partial y}{\partial x}-\phi\right)=0, \\
I_{h} \frac{\partial^{2} \vartheta}{\partial t^{2}}=E I \frac{\partial \phi(t, 0)}{\partial x}+u, \\
m \frac{\partial^{2}}{\partial t^{2}}(y(L, t)+L \vartheta)=-\kappa G A\left(\frac{\partial y(L, t)}{\partial x}-\phi(L, t)\right), \\
I_{c} \frac{\partial^{2}}{\partial t^{2}}(\phi(L, t)+\vartheta)=-E I \frac{\partial \phi(L, t)}{\partial x},
\end{gathered}
$$




$$
\begin{aligned}
& y(0, t)=0, \\
& \phi(0, t)=0 .
\end{aligned}
$$

Taking the Laplace transform of (10)-(16), we get

$$
\begin{gathered}
\rho A s^{2}(Y+x \Theta)-\kappa G A\left(\frac{\partial^{2} Y}{\partial x^{2}}-\frac{\partial \Theta}{\partial x}\right)=0 \\
\rho I s^{2}(\Phi+\Theta)-E I \frac{\partial^{2} \Phi}{\partial x^{2}}-\kappa G A\left(\frac{\partial Y}{\partial x}-\Phi\right)=0 \\
I_{h} s^{2} \Theta=E I \frac{\partial \Phi(s, 0)}{\partial x}+U \\
m s^{2}(Y(l, s)+L \Theta)=-\kappa G A\left(\frac{\partial y(L, s)}{\partial x}-\Phi(L, s)\right) \\
I_{c} s^{2}(\Phi(L, s)+\Theta)=-E I \frac{\partial \Phi(L, s)}{\partial x} \\
Y(0, s)=0 \\
\Phi(0, t)=0
\end{gathered}
$$

\section{Stabilizing Feedback Controls}

3.1. Translational Arm. The objective is to derive explicit expressions for feedback control $u$ for controlling the arm. The approach taken here is to consider the total energy of the arm at any time $t$ given by

$$
\begin{aligned}
V= & \frac{1}{2} \int_{0}^{L}\left[\rho A\left\{\frac{\partial y}{\partial t}+\frac{\partial w_{b}}{\partial t}\right\}^{2}+\rho I\left\{\frac{\partial \phi}{\partial t}\right\}^{2}\right] d x \\
& +\frac{1}{2} M_{p}\left\{\frac{\partial w_{b}}{\partial t}\right\}^{2} \\
& +\frac{1}{2} \int_{0}^{L}\left[E I\left\{\frac{\partial \phi}{\partial x}\right\}^{2}+\kappa G A\left\{\phi-\frac{\partial y}{\partial x}\right\}^{2}\right] d x,
\end{aligned}
$$

that is positive definite with respect to $y=0, d y / d t=0$.

The Time derivative of (24) is given by

$$
\begin{aligned}
& \frac{\partial V}{\partial t}=\int_{0}^{L}\left[\rho A\left\{\frac{\partial y}{\partial t}+\frac{\partial w_{b}}{\partial t}\right\}\left\{\frac{\partial^{2} y}{\partial t^{2}}+\frac{\partial^{2} w_{b}}{\partial t^{2}}\right\}\right. \\
&\left.+\rho I\left\{\frac{\partial \phi}{\partial t}\right\}\left\{\frac{\partial^{2} \phi}{\partial t^{2}}\right\}\right] d x \\
&+ M_{p}\left\{\frac{\partial w_{b}}{\partial t}\right\}\left\{\frac{\partial^{2} w_{b}}{\partial t^{2}}\right\} \\
&+ \int_{0}^{L}\left[E I\left\{\frac{\partial \phi}{\partial x}\right\}\left\{\frac{\partial^{2} \phi}{\partial x \partial t}\right\}\right. \\
&\left.+\kappa G A\left\{\phi-\frac{\partial y}{\partial x}\right\}\left\{\frac{\partial \phi}{\partial t}-\frac{\partial^{2} y}{\partial x \partial t}\right\}\right] d x .
\end{aligned}
$$

By substituting (1) reduces to

$$
\begin{aligned}
\frac{\partial V}{\partial t}= & \int_{0}^{L}\left[\left\{\frac{\partial y}{\partial t}+\frac{\partial w_{b}}{\partial t}\right\}\left\{\kappa G A\left(\frac{\partial^{2} y}{\partial x^{2}}-\frac{\partial \phi}{\partial x}\right)\right\}\right. \\
& \left.+\left\{\frac{\partial \phi}{\partial t}\right\}\left\{E I \frac{\partial^{2} \phi}{\partial x^{2}}-\kappa G A\left(\phi-\frac{\partial y}{\partial x}\right)\right\}\right] d x \\
+ & \frac{\partial w_{b}}{\partial t}\left\{\kappa G A\left(\phi(0, t)-\frac{\partial y(0, t)}{\partial x}\right)+u\right\} \\
+ & \int_{0}^{L}\left[E I\left\{\frac{\partial \phi}{\partial x}\right\}\left\{\frac{\partial^{2} \phi}{\partial x \partial t}\right\}\right. \\
& \left.+\kappa G A\left\{\phi(x, t)-\frac{\partial y}{\partial x}\right\}\left\{\frac{\partial \phi}{\partial t}-\frac{\partial^{2} y}{\partial x \partial t}\right\}\right] d x .
\end{aligned}
$$

We wish to derive stabilizing feedback controls $u$ such that the resulting feedback-controlled arm is dissipative and stable

$$
\frac{\partial V}{\partial x}=\frac{\partial w_{b}}{\partial t} u
$$

Since our goal is to design a stabilizing control input, the most natural choice is to make the time derivative of the Lyapunov function negative in such a way. The following stabilizing feedback control is chosen:

$$
u=-\gamma \frac{\partial w_{b}}{\partial t} \quad(\gamma>0)
$$

which results in

$$
\frac{\partial V}{\partial t}=-\gamma\left(\frac{\partial w_{b}}{\partial t}\right)^{2} \leq 0
$$

This means that a control input should be chosen with as large as possible and should generate a base velocity that ensures asymptotic stability of the tip bending motion of the arm.

3.2. Rotational Arm. The problem is to find feedback control $u$ such that the arm's total energy given by

$$
\begin{aligned}
V= & \frac{1}{2} \int_{0}^{L}\left[\rho A\left\{\frac{\partial y}{\partial t}+x \frac{\partial \theta}{\partial t}\right\}^{2}+\rho I\left\{\frac{\partial \phi}{\partial t}+\frac{\partial \theta}{\partial t}\right\}^{2}\right] d x \\
& +\frac{1}{2} I_{h}\left\{\frac{\partial \theta}{\partial t}\right\}^{2} \\
& +\frac{1}{2} \int_{0}^{L}\left[E I\left\{\frac{\partial \phi}{\partial x}\right\}^{2}+\kappa G A\left\{\phi-\frac{\partial y}{\partial x}\right\}^{2}\right] d x
\end{aligned}
$$


The time rate-of-change of $V$ is given by

$$
\begin{aligned}
& \frac{\partial V}{\partial t} \int_{0}^{L}\left[\rho A\left\{\frac{\partial y}{\partial t}+x \frac{\partial \theta}{\partial t}\right\}\left\{\frac{\partial^{2} y}{\partial t^{2}}+x \frac{\partial^{2} \theta}{\partial t^{2}}\right\}\right. \\
&\left.+\rho I\left\{\frac{\partial \phi}{\partial t}+\frac{\partial \theta}{\partial t}\right\}\left\{\frac{\partial^{2} \phi}{\partial x^{2}}+\frac{\partial^{2} \theta}{\partial t^{2}}\right\}\right] d x \\
&+I_{h}\left\{\frac{\partial \theta}{\partial t}\right\}\left\{\frac{\partial^{2} \theta}{\partial t^{2}}\right\} \\
&+\int_{0}^{L}\left[E I\left\{\frac{\partial \phi}{\partial x}\right\}\left\{\frac{\partial^{2} \phi}{\partial x \partial t}\right\}\right. \\
&\left.+\kappa G A\left\{\phi-\frac{\partial y}{\partial x}\right\}\left\{\frac{\partial \phi}{\partial t}-\frac{\partial^{2} y}{\partial x \partial t}\right\}\right] d x .
\end{aligned}
$$

By substituting (10)-(11) reduces to

$$
\begin{aligned}
& \frac{\partial V}{\partial t}= \int_{0}^{L}\left[\left\{\frac{\partial y}{\partial t}+x \frac{\partial \theta}{\partial t}\right\}\left\{\kappa G A\left(\frac{\partial^{2} y}{\partial x^{2}}-\frac{\partial \phi}{\partial x}\right)\right\}\right. \\
&\left.+\left\{\frac{\partial \phi}{\partial t}+\frac{\partial \theta}{\partial x}\right\}\left\{E I \frac{\partial^{2} \phi}{\partial x^{2}}-\kappa G A\left(\phi-\frac{\partial y}{\partial x}\right)\right\}\right] d x \\
&+ \frac{\partial \theta}{\partial t}\left\{E I \frac{\partial \phi(0, t)}{\partial x}+u\right\} \\
&+ \int_{0}^{L}\left[E I\left\{\frac{\partial \phi}{\partial x}\right\}\left\{\frac{\partial^{2} \phi}{\partial x \partial t}\right\}\right. \\
&\left.+\kappa G A\left\{\phi(x, t)-\frac{\partial y}{\partial x}\right\}\left\{\frac{\partial \phi}{\partial t}-\frac{\partial^{2} y}{\partial x \partial t}\right\}\right] d x .
\end{aligned}
$$

The equation (32) is then integrated by parts and boundary conditions, (12)-(16) are substituted into the resulting equation. The result is

$$
\frac{\partial V}{\partial t}=\frac{\partial \theta}{\partial t} u
$$

Taking into consideration of the fact that there is a feedback control law which gives nonpositive $\partial V / \partial t$ is

$$
u=-\gamma \frac{\partial \theta}{\partial t} \quad(\gamma>0)
$$

Substituting (34) into (33) leads to

$$
\frac{\partial V}{\partial t}=-\gamma\left(\frac{\partial \theta}{\partial t}\right)^{2} \leq 0
$$

which is negative semidefinite. This implies that the closedloop system is energy dissipative and, hence, stable.

\section{Passivity of Timoshenko Arm}

Taking the time derivative of the total energy, and then integrating from 0 to $t$, we get the following restatement of energy balance principle:

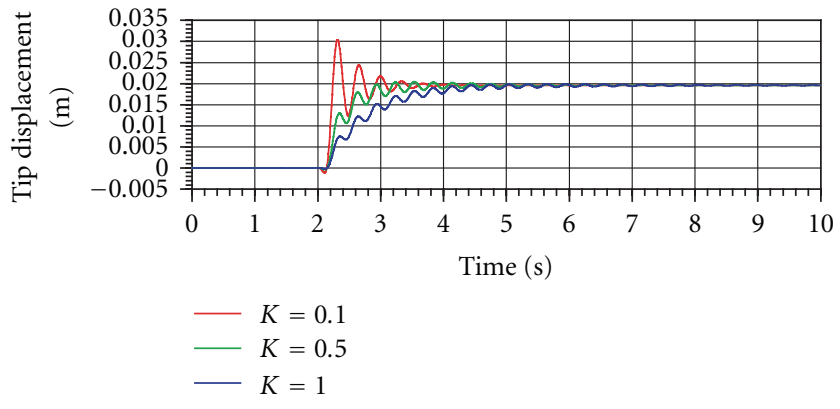

FIgure 3: Step response of the translational Euler arm.

(1) translational arm

$\int_{0}^{t} \frac{\partial w_{b}}{\partial \tau} u d \tau=\int_{0}^{t} \frac{\partial V}{\partial \tau} d \tau=V(t)-V(0) \geq-V(0)=-\gamma_{0}^{2}$

(2) rotational arm

$$
\int_{0}^{t} \frac{\partial \theta}{\partial \tau} u d \tau=\int_{0}^{t} \frac{\partial V}{\partial \tau} d \tau=V(t)-V(0) \geq-V(0)=-\gamma_{0}{ }^{2}
$$

where the left-hand side is the supplied energy and righthand side is the energy at time $t$ minus the initial energy. Passivity of the operator $u$ relative to the function $V$ follows from the equation above and positivity of the total energy.

\section{Simulation Results}

Numerical inversion of Laplace transform is used to obtain the results in the time domain. The computation of the inverse Laplace transform is based on the paper of Hosono [14]. Numerical solutions are obtained by the Bromwich integrating the system corresponding to (A.1) and so forth. In the computer simulation study, we consider a typical arm whose parameters are given in the Table 1. Figures 3-6 show the step response of the arm-tip displacement with feedback gains variations. Figures 3 and 5 show the results of the Euler arm. Figures 4 and 6 show the results of the Timoshenko arm. There are few differences with each other in the case of this system parameters. It can be seen that the arm-tip displacement toward the desired position. With increasing the feedback gains, the settling times are longer and the residual vibrations are more slightly damped. In this case, shear deformations are small and negligible, so that the shear angle is almost zero and the rotary inertia is small and negligible, the so-called Euler-Bernoulli beam equations result. If the beam is long and skinny (aspect ratio, say greater than 10), the Euler-Bernoulli assumptions are appropriate.

\section{Conclusion}

In this paper, the passivity-based control of rotational and translational Timoshenko arms is studied. This approach has 


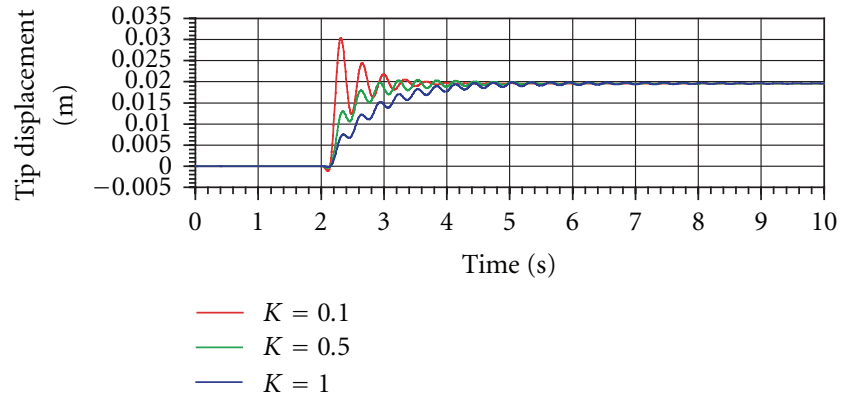

Figure 4: Step response of the translational Timoshenko arm.

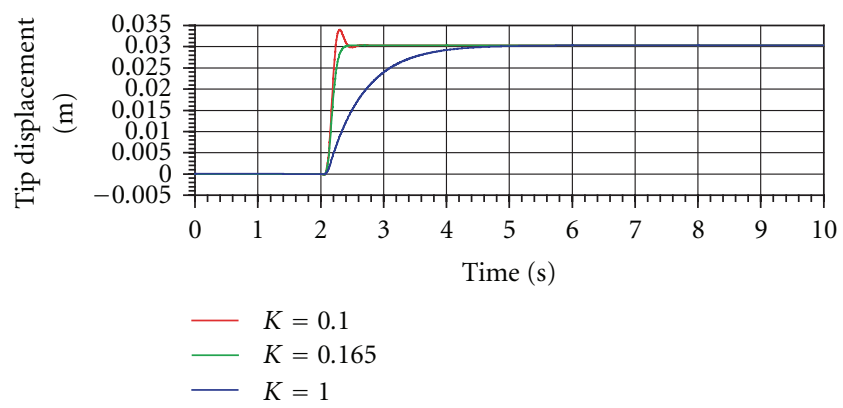

FIgURE 5: Step response of the rotational Euler arm.

the advantage over the conventional methods in the respect that it allows one to deal directly with the system's partial differential equations without resorting to approximations. Numerical results for the control of a translational and rotational flexible Timoshenko arm are presented and compared in graphical plots. They verify that the proposed control schemes are effective at controlling flexible dynamical systems and vibration control of mechanical systems.

\section{Appendix}

In the case of the translational Timoshenko arm, the resulting solution to (3), (4), and (5) is given by

$$
Y(x, s)=\frac{\Delta k_{1}}{\Delta} e^{\lambda_{1} x}+\frac{\Delta k_{2}}{\Delta} e^{\lambda_{2} x}-\frac{\Delta k_{3}}{\Delta} e^{-\lambda_{1} x}-\frac{\Delta k_{4}}{\Delta} e^{-\lambda_{2} x}-W_{b},
$$

where

$$
\begin{aligned}
& a_{11}=\left\{m s^{2}+\frac{\rho A s^{2}}{\lambda_{1}}\right\} e^{\lambda_{1} L}, \\
& a_{12}=\left\{m s^{2}+\frac{\rho A s^{2}}{\lambda_{2}}\right\} e^{\lambda_{2} L}, \\
& a_{13}=\left\{m s^{2}-\frac{\rho A s^{2}}{\lambda_{1}}\right\} e^{-\lambda_{1} L}, \\
& a_{14}=\left\{m s^{2}-\frac{\rho A s^{2}}{\lambda_{2}}\right\} e^{-\lambda_{2} L},
\end{aligned}
$$

TABLE 1: System parameters.

\begin{tabular}{lccc}
\hline Parameter & Nomenclature & Values & Unit \\
\hline $\begin{array}{l}\text { Length of the arm } \\
\begin{array}{l}\text { Arm cross-sectional } \\
\text { area }\end{array}\end{array}$ & $L$ & 1.2 & $\mathrm{~m}$ \\
$\begin{array}{l}\text { Volume mass } \\
\text { density }\end{array}$ & $\rho$ & $2.5 \times 10^{-4}$ & $\mathrm{~m}^{2}$ \\
$\begin{array}{l}\text { Young's modulus } \\
\text { Internal structural }\end{array}$ & $E$ & $6.8944 \times 10^{10}$ & $\mathrm{~Pa}$ \\
$\begin{array}{l}\text { damping } \\
\begin{array}{l}\text { Payload moment of } \\
\text { inertia }\end{array}\end{array}$ & $C$ & 0 & $\mathrm{Kg} / \mathrm{sec}$ \\
$\begin{array}{l}\text { Payload mass } \\
\text { Shear correction } \\
\text { factor }\end{array}$ & $I_{c}$ & $5 \times 10^{-4}$ & $\mathrm{Kg} \cdot \mathrm{m}^{2}$ \\
$\begin{array}{l}\text { Shear modulus } \\
\text { Slider mass }\end{array}$ & $\kappa$ & 0.2 & $\mathrm{Kg}$ \\
$\begin{array}{l}\text { Hub moment of } \\
\text { inertia }\end{array}$ & $M_{p}$ & $2.6517 \times 10^{10}$ & $\mathrm{~Pa}$ \\
\hline
\end{tabular}

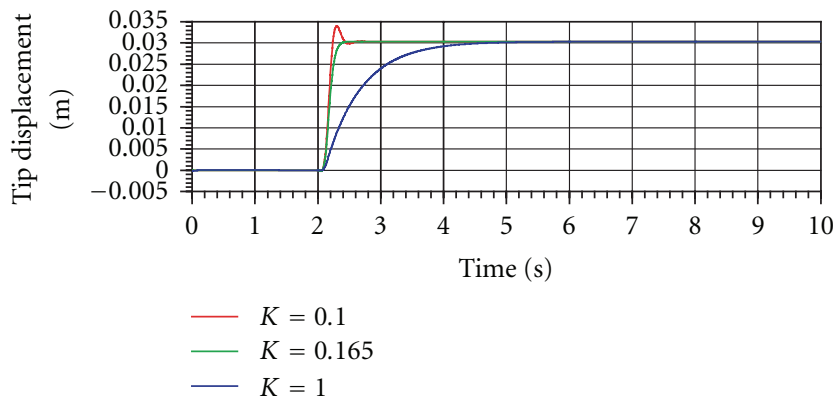

FIgURE 6: Step response of the rotational Timoshenko arm.

$$
\begin{aligned}
& a_{21}=\left(\lambda_{1}-\frac{\rho s^{2}}{\kappa G \lambda_{1}}\right)\left(I_{c} s^{2}+E I(1+C s) \lambda_{1}\right) e^{\lambda_{1} L}, \\
& a_{22}=\left(\lambda_{2}-\frac{\rho s^{2}}{\kappa G \lambda_{2}}\right)\left(I_{c} s^{2}+E I(1+C s) \lambda_{2}\right) e^{\lambda_{2} L}, \\
& a_{23}=-\left(\lambda_{1}-\frac{\rho s^{2}}{\kappa G \lambda_{1}}\right)\left(I_{c} s^{2}-E I(1+C s) \lambda_{1}\right) e^{-\lambda_{1} L}, \\
& a_{24}=-\left(\lambda_{2}-\frac{\rho s^{2}}{\kappa G \lambda_{2}}\right)\left(I_{c} s^{2}-E I(1+C s) \lambda_{2}\right) e^{-\lambda_{2} L}, \\
& a_{31}=\left(\lambda_{1}-\frac{\rho s^{2}}{\kappa G \lambda_{1}}\right), \\
& a_{32}=\left(\lambda_{2}-\frac{\rho s^{2}}{\kappa G \lambda_{2}}\right), \\
& a_{33}=-\left(\lambda_{1}-\frac{\rho s^{2}}{\kappa G \lambda_{1}}\right), \\
& a_{34}=-\left(\lambda_{2}-\frac{\rho s^{2}}{\kappa G \lambda_{2}}\right),
\end{aligned}
$$




$$
\begin{aligned}
& a_{41}=\left(M_{p} s^{2}-\frac{\rho A s^{2}}{\lambda_{1}}\right), \\
& a_{42}=\left(M_{p} s^{2}-\frac{\rho A s^{2}}{\lambda_{2}}\right), \\
& a_{43}=\left(M_{p} s^{2}+\frac{\rho A s^{2}}{\lambda_{1}}\right), \\
& a_{44}=\left(M_{p} s^{2}+\frac{\rho A s^{2}}{\lambda_{2}}\right),
\end{aligned}
$$

$$
\Delta=\left[\begin{array}{llll}
a_{11} & a_{12} & a_{13} & a_{14} \\
a_{21} & a_{22} & a_{23} & a_{24} \\
a_{31} & a_{32} & a_{33} & a_{34} \\
a_{41} & a_{42} & a_{43} & a_{44}
\end{array}\right],
$$$$
\Delta k_{1}=\left[\begin{array}{cccc}
0 & a_{12} & a_{13} & a_{14} \\
0 & a_{22} & a_{23} & a_{24} \\
0 & a_{32} & a_{33} & a_{34} \\
U & a_{42} & a_{43} & a_{44}
\end{array}\right] \text {, }
$$$$
\Delta k_{2}=\left[\begin{array}{llll}
a_{11} & 0 & a_{13} & a_{14} \\
a_{21} & 0 & a_{23} & a_{24} \\
a_{31} & 0 & a_{33} & a_{34} \\
a_{41} & U & a_{43} & a_{44}
\end{array}\right],
$$$$
\Delta k_{3}=\left[\begin{array}{llll}
a_{11} & a_{12} & 0 & a_{14} \\
a_{21} & a_{22} & 0 & a_{24} \\
a_{31} & a_{32} & 0 & a_{34} \\
a_{41} & a_{42} & U & a_{44}
\end{array}\right],
$$$$
\Delta k_{4}=\left[\begin{array}{llll}
a_{11} & a_{12} & a_{13} & 0 \\
a_{21} & a_{22} & a_{23} & 0 \\
a_{31} & a_{32} & a_{33} & 0 \\
a_{41} & a_{42} & a_{43} & U
\end{array}\right],
$$$$
\lambda= \pm\left[\frac { 1 } { 2 } \left\{\left(\frac{1}{E(1+C s)}+\frac{1}{\kappa G}\right) \rho s^{2}\right.\right.
$$

$$
\begin{aligned}
& \pm\left(\frac{\rho^{2} s^{4}}{E^{2}(1+C s)^{2}}-\frac{2 \rho^{2} s^{4}}{E(1+C s) \kappa G}\right. \\
& \left.\left.\left.+\frac{\rho^{2} s^{4}}{\kappa^{2} G^{2}}-\frac{4 \rho A s^{2}}{E I(1+C s)}\right)^{1 / 2}\right\}\right]^{1 / 2}, \lambda= \pm \lambda_{1}, \pm \lambda_{2} .
\end{aligned}
$$

In the case of the rotational Timoshenko arm, the resulting solution to (17), (18), and (19) can be obtained (omitted details for want of space).

\section{References}

[1] K. Yuan and C. M. Hu, "Nonlinear modeling and partial linearizing control of a slewing timoshenko-beam," Journal of Dynamic Systems, Measurement and Control, Transactions of the ASME, vol. 118, no. 1, pp. 75-82, 1996.

[2] M. Tadi, "Comparison of two finite-element schemes for feedback control of a Timoshenko beam," in Proceedings of the ASME Dynamic Systems and Control Division (DSC '97), vol. 61, pp. 587-596, November 1997.

[3] M. W. D. White and G. R. Heppler, "Vibration of a rotating timoshenko beam," Journal of Vibration and Acoustics, Transactions of the ASME, vol. 118, no. 4, pp. 606-613, 1996.

[4] M. J. Balas, "Direct velocity feedback control of large space structures," Journal of Guidance, Control, and Dynamics, vol. 2, no. 3, pp. 252-253, 1979.

[5] Z. H. Luo, "Direct strain feedback control of flexible robot arms: new theoretical and experimental results," IEEE Transactions on Automatic Control, vol. 38, no. 11, pp. 1610-1622, 1993.

[6] B. Paden, D. Chen, R. Ledesma, and E. Bayo, "Exponentially stable tracking control for multijoint flexible-link manipulators," Journal of Dynamic Systems, Measurement and Control, Transactions of the ASME, vol. 115, no. 1, pp. 53-59, 1993.

[7] M. Karkoub and K. Tamma, "Modelling and $\mu$-synthesis control of flexible manipulators," Computers and Structures, vol. 79, no. 5, pp. 543-551, 2001.

[8] S. K. Dwivedy and P. Eberhard, "Dynamic analysis of flexible manipulators, a literature review," Mechanism and Machine Theory, vol. 41, no. 7, pp. 749-777, 2006.

[9] G. G. Rigatos, "Model-based and model-free control of flexible-link robots: a comparison between representative methods," Applied Mathematical Modelling, vol. 33, no. 10, pp. 3906-3925, 2009.

[10] C. Di Castri and A. Messina, "Vibration analysis of a planar multilink manipulator using a reduced-order matrix formulation," Mechanics Research Communications, vol. 38, no. 3, pp. 231-238, 2011.

[11] J. J. Shifman, "Lyapunov functions and the control of the Euler-Bernoulli beam," International Journal of Control, vol. 57, no. 4, pp. 971-990, 1993.

[12] J. LaSalle and S. Lefschetz, Stability by Lyapunov's Direct Method with Applications, Academic Press, New York, NY, USA, 1961.

[13] R. Ortega, A. Loria, P. J. Nicklasson, and H. Sira-Ramirez, Passivity-Based Control of Euler-Lagrange Systems, Springer, London, UK, 1998.

[14] T. Hosono, "Numerical inversion of Laplace transform and some application to wave optics," Radio Science, vol. 16, no. 6, pp. 1015-1019, 1980. 

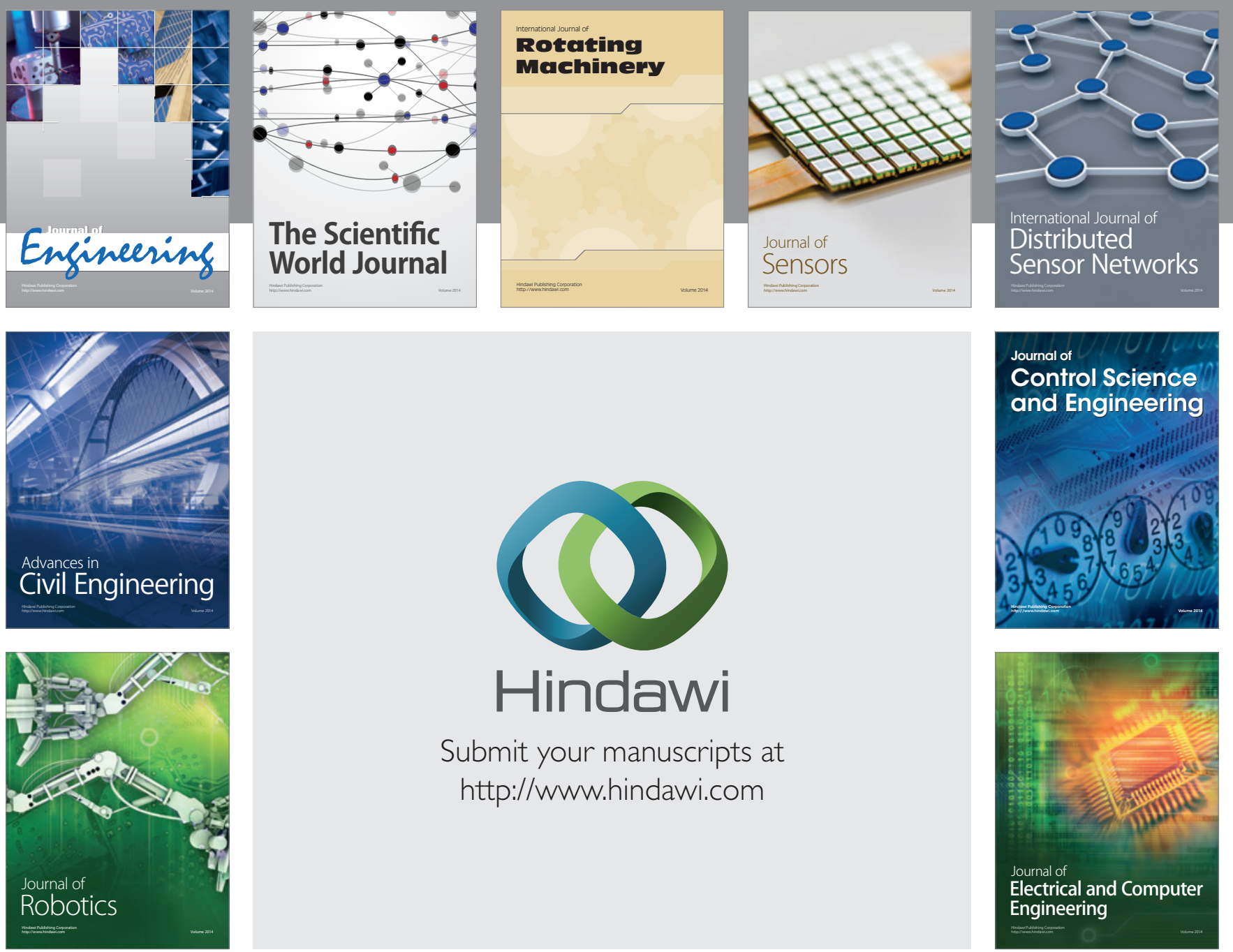

Submit your manuscripts at

http://www.hindawi.com
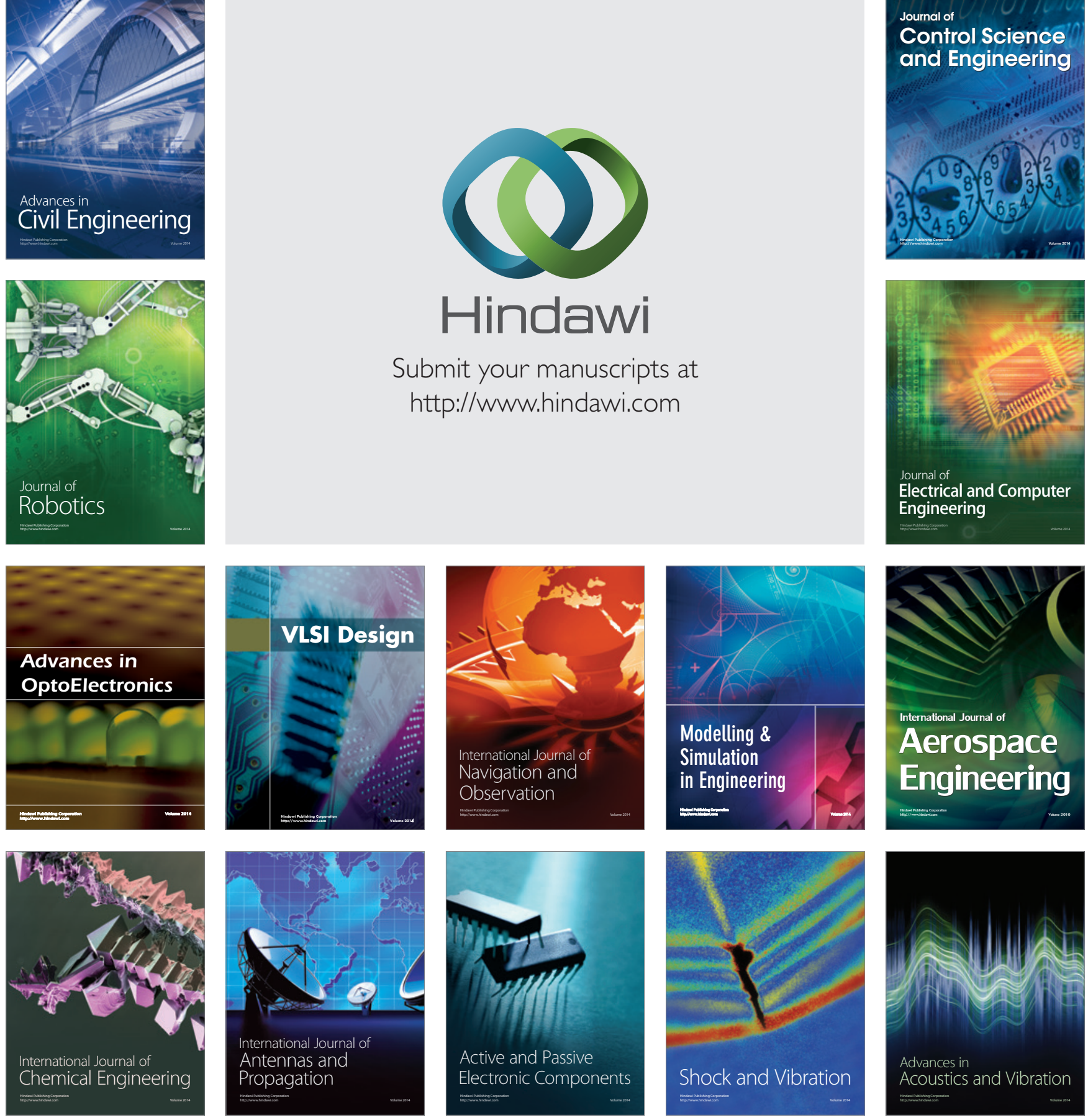